Pacific

Journal of

Mathematics

TOPOLOGY VERSUS CHERN NUMBERS

FOR COMPLEX 3-FOLDS

Claude LeBrun

Volume $191 \quad$ No. 1

November 1999 


\title{
TOPOLOGY VERSUS CHERN NUMBERS FOR COMPLEX 3-FOLDS
}

\author{
Claude LeBrun
}

\begin{abstract}
We show by example that the Chern numbers $c_{1}^{3}$ and $c_{1} c_{2}$ of a complex 3-fold are not determined by the topology of the underlying smooth compact 6-manifold. In fact, we observe that infinitely many different values of a Chern number can be achieved by (integrable) complex structures on a fixed 6manifold.
\end{abstract}

\section{Introduction.}

Suppose that $X$ is a smooth compact oriented 6-manifold. Recall that an almost-complex structure on $X$ means an endomorphism $J: T X \rightarrow T X$ of the tangent bundle of $X$ with $J^{2}=-1$ which determines the given orientation of $X$. Such a structure makes $T X$ into a complex vector bundle, so that one can speak of the Chern classes $c_{j} \in H^{2 j}(X, \mathbb{Z})$ of $(X, J)$, and therefore of the Chern numbers

$$
\begin{aligned}
\mathbf{c}_{\mathbf{1}}^{\mathbf{3}} & =\int_{X} c_{1}^{3} \\
\mathbf{c}_{\mathbf{1}} \mathbf{c}_{\mathbf{2}} & =\int_{X} c_{1} c_{2} \\
\mathbf{c}_{\mathbf{3}} & =\int_{X} c_{3}
\end{aligned}
$$

of the almost-complex manifold $(X, J)$. The only obstruction [10] to the existence of an almost-complex structure $J$ on $X$ is that $X$ be $\operatorname{spin}^{c}$. This happens precisely when the second Stiefel-Whitney class $w_{2}(X) \in H^{2}\left(X, \mathbb{Z}_{2}\right)$ can be written as the mod-2 reduction of an element of $H^{2}(X, \mathbb{Z})$, in which case each preimage of $w_{2}$ in $H^{2}(X, \mathbb{Z})$ can be realized as $c_{1}$ for some almostcomplex structure $J$. It follows that the Chern numbers $\mathbf{c}_{\mathbf{1}}^{\mathbf{3}}$ and $\mathbf{c}_{\mathbf{1}} \mathbf{c}_{\mathbf{2}}$ of the almost-complex $(X, J)$ are certainly not topological invariants of the 6-manifold $X$. For example, if $X=\mathbb{C P}_{3}$, every integer of the form $8 j$ can be realized as $\mathbf{c}_{\mathbf{1}} \mathbf{c}_{\mathbf{2}}$, and every integer of the form $8 j^{3}$ can be realized as $\mathbf{c}_{\mathbf{1}}^{\mathbf{3}}$ for some almost-complex structure $J$ on $\mathbb{C P}_{3}$. On the other hand, $c_{3}$ is the Euler class of $T X$, so that $\mathbf{c}_{\mathbf{3}}=\chi(X)$ is actually a homotopy invariant of $X$. 
In this note, we will observe that the above situation persists even if one demands that the almost-complex structures under consideration be integrable. Recall that an almost-complex structure $J$ on $X$ is called a complex structure if it is integrable, in the sense of being locally isomorphic to the standard, constant-coefficient structure on $\mathbb{R}^{6}=\mathbb{C}^{3}$. The question of whether the Chern numbers $\mathbf{c}_{\mathbf{1}}^{\mathbf{3}}$ and $\mathbf{c}_{\mathbf{1}} \mathbf{c}_{\mathbf{2}}$ of a complex 3 -fold might actually be topological invariants of the underlying 6-manifold was raised, for example, in an interesting survey article by Okonek and van de Ven [7, p. 317].

Our principal results are as follows:

Theorem A. There is a compact simply connected 6 -manifold $X$ which admits a sequence $J_{m}, m \in \mathbb{Z}^{+}$, of (integrable) complex structures with

$$
\mathbf{c}_{\mathbf{1}} \mathbf{c}_{\mathbf{2}}\left(X, J_{m}\right)=48 \mathrm{~m} .
$$

Indeed, there are infinitely many homeotypes of $X$ with this property.

Theorem B. Let $(m, n)$ be any pair of integers. Then for any integer $\tilde{n} \ll$ $n$, there is a complex projective 3 -fold $(X, J)$ with Chern numbers

$$
\mathbf{c}_{\mathbf{1}} \mathbf{c}_{\mathbf{2}}=24 m, \mathbf{c}_{\mathbf{1}}^{\mathbf{3}}=8 n,
$$

which admits a second complex structure $\tilde{J}$ with

$$
\mathbf{c}_{\mathbf{1}} \mathbf{c}_{\mathbf{2}}=48 m, \mathbf{c}_{\mathbf{1}}^{\mathbf{3}}=8 \tilde{n} .
$$

\section{Infinitely Many Complex Structures.}

The fact that the Chern classes of a complex 3-fold are not determined by the topology of the underlying 6-manifold was observed long ago by Calabi [2]. While his examples all have vanishing Chern numbers, they nonetheless contain the seeds of a natural class of examples which lead to Theorem A:

Theorem 1. For each positive integer $m$, the 6 -manifold $X=K 3 \times S^{2}$ admits a complex structure $J_{m}$ with

$$
\mathbf{c}_{\mathbf{1}} \mathbf{c}_{\mathbf{2}}\left(X, J_{m}\right)=48 m
$$

and $\mathbf{c}_{\mathbf{1}}^{\mathbf{3}}\left(X, J_{m}\right)=0$.

Proof. Let $M$ denote the underlying oriented 4-manifold of the $K 3$ surface, and let $g$ be any hyper-Kähler metric on $M$; such metrics exist by Yau's solution of the Calabi conjecture $[\mathbf{1 1}]$. Let $Z$ be the twistor space $[\mathbf{1}, \mathbf{8}]$ of $(M, g)$, and let $\varpi: Z \rightarrow \mathbb{C P}_{1}$ be the holomorphic projection induced by the hyper-Kähler structure. Differentiably, $\varpi$ is the trivial fiber bundle with fiber $M$, so that $Z$ may be thought of as $X=M \times S^{2}$ equipped with a complex structure.

Now let $f_{m}: \mathbb{C P}_{1} \rightarrow \mathbb{C P}_{1}$ be a holomorphic map of degree $m-1$; for example, we may take $f_{m}([u, v])=\left[u^{m-1}, v^{m-1}\right]$, where $m$ is any positive 
integer. We may then define a holomorphic family $f_{m}^{*} \varpi$ of $K 3$ 's over $\mathbb{C P}_{1}$ by pulling back the family $\varpi$ via $f_{m}$ :

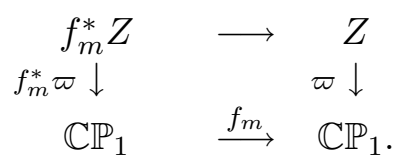

In other words, $f_{m}^{*} Z$ is the inverse image, via

$$
1 \times \varpi: \mathbb{C P}_{1} \times Z \longrightarrow \mathbb{C P}_{1} \times \mathbb{C P}_{1},
$$

of the graph of $f_{m}$. Since $\varpi$ is differentiably trivial, so is $f_{m}^{*} \varpi$, and $f_{m}^{*} Z$ may therefore be viewed as $X=K 3 \times S^{2}$ equipped with a complex structure $J_{m}$.

Now if $\pi: Z \rightarrow M$ is the (non-holomorphic) twistor projection, an explicit diffeomorphism $Z \rightarrow X$ is given by $\pi \times \varpi$, and $f_{m}^{*} Z$ is similarly trivialized by $f_{m}^{*} \varpi \times f_{m}^{*} \pi$. Let $L \subset T f_{m}^{*} Z$ be the kernel of the derivative of the pulled-back twistor projection $f_{m}^{*} \pi$. Then $L$ is $J_{m}$ invariant, despite the fact that $\pi$ is not holomorphic, and so may be viewed as a complex line-bundle. Moreover, $L$ may be identified with the pull-back of the (holomorphic) tangent bundle of $\mathbb{C P}_{1}$ via $f_{m}^{*} \varpi$, so that $c_{1}^{2}(L)=0$, and hence $p_{1}(L)=0$. If, on the other hand, we use $H$ to denote the kernel of the derivative of $f_{m}^{*} \varpi$, then the underlying real bundle of $H$ is $\left(f_{m}^{*} \pi\right)^{*} T M$, and so $p_{1}(H)=f_{m}^{*}\left[p_{1}(M)\right]=-48 F$, where, by Poincaré duality, $F$ is represented by a fiber $S^{2}$ of $f_{m}^{*} \pi$. It follows that $p_{1}\left(f_{m}^{*} Z\right)=p_{1}(L)+p_{1}(H)=-48 F$. On the other hand, any $K 3$ has trivial canonical line bundle, and the fibers of $\pi$ are $\mathbb{C P}_{1}$ 's with normal bundle $\mathcal{O}(1) \oplus \mathcal{O}(1)$, so $c_{1}(H)=c_{1}(L)$ when $m=2$. For general $m$, it follows that $c_{1}(H)=(m-1) c_{1}(L)$, and hence that

$$
c_{1}=m c_{1}(L) .
$$

We therefore have $c_{1}^{2}=m^{2} c_{1}^{2}(L)=0$, so that

$$
\mathbf{c}_{\mathbf{1}} \mathbf{c}_{\mathbf{2}}=\frac{\mathbf{c}_{\mathbf{1}}^{\mathbf{3}}-\mathbf{c}_{\mathbf{1}} \mathbf{p}_{\mathbf{1}}}{2}=24 \int_{F} m c_{1}(L)=48 m,
$$

and $\mathbf{c}_{\mathbf{1}}^{\mathbf{3}}=0$.

When $m=1$, the above complex structure is simply an arbitrary product complex structure on $K 3 \times \mathbb{C P}_{1}$, and so is of Kähler type; indeed, we may even arrange for it to be projective-algebraic if we like. On the other hand, the $m=2$ complex structure is that of a twistor space, and so is never of Kähler type by Hitchin's classification of Kählerian twistor spaces [4]. For large values of $m$, one can prove something even stronger: $J_{m}$ isn't even homotopic to a complex structure of Kähler type. This is because the Todd genus

$$
1-h^{1}(\mathcal{O})+h^{2}(\mathcal{O})-h^{3}(\mathcal{O})=\chi(\mathcal{O})=\frac{\mathbf{c}_{\mathbf{1}} \mathbf{c}_{\mathbf{2}}}{24}=2 m,
$$


so that $h^{2}(\mathcal{O})$ will eventually exceed $b_{2}(X)$, in violation of the Hodge decomposition. In the next section, we will see that this phenomenon is actually quite typical.

In order to show that there is more than one 6-manifold for which infinitely many different values of a Chern number are achieved by (integrable) complex structures, we may now invoke the standard process of blowing up. The following facts about blow-up 3-folds are left as exercises for the reader.

Proposition 2. Let $(X, J)$ be any compact complex 3 -fold, and let $(\hat{X}, \hat{J})$ be obtained from $(X, J)$ by blowing up a point. Then $\hat{X}$ is diffeomorphic to the connected sum $X \# \mathbb{C P}_{3}$, and if $X$ is spin, so is $\hat{X}$. Moreover, the Chern numbers of the blow-up are related to those of the original 3-fold by

$$
\begin{aligned}
\mathbf{c}_{\mathbf{1}}^{\mathbf{3}}(\hat{X}, \hat{J}) & =\mathbf{c}_{\mathbf{1}}^{\mathbf{3}}(X, J)+8 \\
\mathbf{c}_{\mathbf{1}} \mathbf{c}_{\mathbf{2}}(\hat{X}, \hat{J}) & =\mathbf{c}_{\mathbf{1}} \mathbf{c}_{\mathbf{2}}(X, J) \\
\mathbf{c}_{\boldsymbol{3}}(\hat{X}, \hat{J}) & =\mathbf{c}_{\mathbf{3}}(X, J)+2 .
\end{aligned}
$$

Iterated blow-ups of the previous examples thus prove the following precise form of Theorem A:

Corollary 3. For each integer $n \geq 0$, the 6-dimensional spin manifold

$$
X=\left(K 3 \times S^{2}\right) \# n \mathbb{C P}_{3}
$$

admits a sequence $J_{m}$ of complex structures with

$$
\begin{aligned}
\mathbf{c}_{\mathbf{1}} \mathbf{c}_{\mathbf{2}}\left(X, J_{m}\right) & =48 m \\
\mathbf{c}_{\mathbf{1}}^{\mathbf{3}}\left(X, J_{m}\right) & =8 n .
\end{aligned}
$$

\section{Kähler Type.}

We saw in Theorem 1 that it is possible to find 6-manifolds with sequences of complex structures for which a Chern number takes on infinitely many different values. On the other hand, if one requires that the complex structures in question be of Kähler type, one arrives at essentially the opposite conclusion. This is illustrated by our next result.

Theorem 4. Let $X$ be the underlying compact oriented 6-manifold of any Kählerian 3-fold. Then there exist infinitely many homotopy classes of almost-complex structures on $X$ which cannot be represented by complex structures of Kähler type.

Proof. By assumption, there is a Kähler class $[\omega] \in H^{2}(X, \mathbb{R})$ with $[\omega]^{3} \neq 0$. Now $H^{2}(X, \mathbb{Q}) \subset H^{2}(X, \mathbb{R})$ is dense, and the cup form is continuous, so approximating $[\omega]$ with rational classes will produce classes $\alpha_{0} \in H^{2}(X, \mathbb{Q})$ with $\alpha_{0}^{3}>0$. Multiplying by a suitable positive integer $k$ to clear denominators, we may thus obtain a class $k \alpha_{0}$ which is the image of an integer 
class $\alpha \in H^{2}(X, \mathbb{Z})$ in rational cohomology. Now let $\beta \in H^{2}(X, \mathbb{Z})$ be the first Chern class of the given complex structure on $X$. Then $2 n \alpha+\beta$ is an integer lift of $w_{2}$, and so [10] can be realized as $c_{1}$ for some homotopy class $\left[J_{n}\right]$ of almost-complex structures. Now if $J_{n} \in\left[J_{n}\right]$ is integrable, the Todd genus of $\left(X, J_{n}\right)$ is

$$
\chi(\mathcal{O})=\frac{c_{1} \cdot c_{2}}{24}=\frac{(2 n \alpha+\beta)^{3}-(2 n \alpha+\beta) \cdot p_{1}}{48}
$$

which is cubic in $n$, with the coefficient of $n^{3}$ non-vanishing. It therefore follows that there is an integer $n_{0}$ such that $\left|\sum_{k}(-1)^{k} h^{0, k}\right|>\sum_{j} b_{j}(X)$ whenever $|n|>n_{0}$. For $n$ in this range, the Hodge theorem must therefore fail, and so an integrable $J_{n}$ could not possibly be of Kähler type.

The reader should note that we have only used two mild consequences of the Kähler condition: the degeneration of the Fröhlicher spectral sequence, and the non-triviality of the cup form on $H^{2}$. The same argument would thus apply if one instead wished to consider, say, complex structures of Moishezon type.

\section{Independence of Chern Numbers.}

So far, we have seen that the Chern number $\mathbf{c}_{\mathbf{1}} \mathbf{c}_{\mathbf{2}}$ of a complex 3 -fold is not an invariant of the underlying 6 -manifold. We will now see see that the same is true of $\mathbf{c}_{\mathbf{1}}^{\mathbf{3}}$.

To this end, let $N$ be any smooth, compact oriented 4-manifold. By [9], the connected sum $M=N \# k \overline{\mathbb{C P}}_{2}$ admits anti-self-dual metrics $g$ provided that $k$ is sufficiently large. The twistor space of such an anti-self-dual metric is a complex 3 -fold $\left(Z, J_{2}\right)$, the underlying 6 -manifold $Z$ of which is formally the fiber-wise projectivization $\mathbb{P}\left(\mathbb{S}_{+}\right)$of the bundle of positive spinors on $M$. This description may seem a bit paradoxical, insofar as we are interested in choices of $M$ which definitely are not spin, but it may be made quite concrete by choosing a $\operatorname{spin}^{c}$ structure on $M$. This then gives rise to a well defined "twisted spinor" bundle $V_{+}$which formally satisfies

$$
V_{+}=\mathbb{S}_{+} \otimes L^{1 / 2}
$$

for a line bundle $L$ with $c_{1}(L) \cong w_{2}(M) \bmod 2$. This done, we then have a canonical identification of $Z$ with the total space of the $\mathbb{C P}_{1}$-bundle $\mathbb{P}\left(V_{+}\right)$. The naturally defined complex structure $J_{2}$ then makes each fiber of the projection $\pi: Z \rightarrow M$ into a holomorphically embedded $\mathbb{C P}_{1}$ with normal bundle $\mathcal{O}(1) \oplus \mathcal{O}(1)$. For more details, see $[\mathbf{1}, \mathbf{4}, \mathbf{8}]$.

Now let us now specialize to the case in which $N$ is a complex surface, and notice that $M=N \# k \overline{\mathbb{C P}}_{2}$ may then be thought of as an iterated blow-up of $N$, and so, in particular, carries a complex structure. This complex structure induces a $\operatorname{spin}^{c}$ structure on $M$ such that, for any metric $g$, the associated 
twisted spin bundle $V_{+}$is smoothly bundle-isomorphic to the holomorphic vector $\mathcal{O} \oplus K^{-1}$, where $K$ is the canonical line bundle of $M$. Indeed, for a Hermitian metric on $M$, there is even a canonical isomorphism $V_{+} \cong \mathcal{O} \oplus$ $K^{-1}$; and, up to abstract the bundle equivalence, the twisted spinor bundle $V_{+}$is metric-independent once a $\operatorname{spin}^{c}$ structure is specified. In this way, the twistor space $Z$ of an anti-self-dual metric $g$ on $M$ is diffeomorphic to the complex manifold $\mathbb{P}\left(\mathcal{O} \oplus K^{-1}\right)$, and so carries a second complex structure $J_{1}$. Notice that we do not need to assume any compatibility between the metric $g$ and the complex structure of $M$. Also notice that $\left(Z, J_{1}\right)$ is projective algebraic (respectively, Kählerian) if $M$ is.

Let us now calculate the Chern numbers of $\left(Z, J_{1}\right)$. To do this, first notice that $Z=\mathbb{P}\left(\mathcal{O} \oplus K^{-1}\right)$ carries two canonical hypersurfaces, $\Sigma$ and $\bar{\Sigma}$, corresponding to the factors of the direct sum $\mathcal{O} \oplus K^{-1}$. These are both copies of the complex surface $M$, but their normal bundles are respectively $K^{-1}$ and $K$. Moreover, the divisor $\Sigma+\bar{\Sigma}$ precisely represents the vertical line bundle $L$ of $Z$. We thus have

$$
c_{1}\left(Z, J_{1}\right)=\Sigma+\bar{\Sigma}+\pi^{*} c_{1}(M)=2 \Sigma .
$$

Hence

$$
\mathbf{c}_{\mathbf{1}}^{\mathbf{3}}\left(Z, J_{1}\right)=(2 \Sigma)^{3}=8(2 \chi+3 \tau)
$$

and

$$
\mathbf{c}_{1} \mathbf{c}_{2}\left(Z, J_{1}\right)=2\left(\mathbf{c}_{\mathbf{1}}^{\mathbf{2}}+\mathbf{c}_{\mathbf{2}}\right)(M)=6(\chi+\tau),
$$

where $\chi$ and $\tau$ are the Euler characteristic and signature of $M$, respectively.

On the other hand, there is a fiber-wise antipodal map which acts antiholomorphically on $\left(Z, J_{2}\right)$, so $c_{1}\left(J_{2}\right)$ is Poincaré dual to an element of $H_{4}(Z)$ which is invariant under this antipodal map. We also know that the integral of $c_{1}\left(J_{2}\right)$ on a fiber is 4 . It follows that

$$
c_{1}\left(Z, J_{2}\right)=2 \Sigma+2 \bar{\Sigma} .
$$

Since the restrictions of $J_{1}$ and $J_{2}$ to a tubular neighborhood of $\Sigma$ are also homotopic, we therefore deduce the formulæ

$$
\begin{aligned}
\mathbf{c}_{\mathbf{1}}^{\mathbf{3}}\left(Z, J_{2}\right) & =16(2 \chi+3 \tau) \\
\mathbf{c}_{1} \mathbf{c}_{\mathbf{2}}\left(Z, J_{2}\right) & =12(\chi+\tau)
\end{aligned}
$$

derived (with opposite orientation conventions) by Hitchin [4] in greater generality. For us, the point is that the invariants $\mathbf{c}_{\mathbf{1}}^{\mathbf{3}}$ and $\mathbf{c}_{\mathbf{1}} \mathbf{c}_{\mathbf{2}}$ of $\left(Z, J_{2}\right)$ are precisely double the corresponding Chern numbers of $\left(Z, J_{1}\right)$.

We are now in a position to prove a more precise version of Theorem B. 
Theorem 5. Let $(m, n)$ be any pair of integers. Then for any integer $\tilde{n} \ll$ $n$, there is a spin, complex projective 3 -fold $(X, J)$ with Chern numbers

$$
\mathbf{c}_{\mathbf{1}} \mathbf{c}_{\mathbf{2}}=24 m, \mathbf{c}_{\mathbf{1}}^{\mathbf{3}}=8 n,
$$

which admits a second complex structure $\tilde{J}$ with

$$
\mathbf{c}_{\mathbf{1}} \mathbf{c}_{\mathbf{2}}=48 m, \mathbf{c}_{\mathbf{1}}^{\mathbf{3}}=8 \tilde{n} .
$$

If $m>0$, moreover, we may even arrange for $X$ to be simply connected.

Proof. For each integer $m$, we begin by choosing a complex algebraic surface $N_{m}$ with Todd genus $(\chi+\tau) / 4=m$. For example, if $m \leq 1$, let us take $N$ to be $C \times \mathbb{C P}_{1}$, where $C$ is a Riemann surface of genus $1-m$. On the other hand, if $m>1$, we may let $N$ be the minimal resolution of $(E \times C) / \mathbb{Z}_{2}$, where $E$ is an elliptic curve, $C$ is a hyperelliptic curve of genus $m-1$, and $\mathbb{Z}_{2}$ acts simultaneously on both factors by the Weierstrass involution. Notice that our choice of $N_{m}$ is simply connected when $m>0$, and that, incidentally, this is the best one can do in principal.

Now, for each $m$, let $k_{0}(m)$ be chosen so that $N_{m} \# k \overline{\mathbb{C P}}_{2}$ admits anti-selfdual metrics for each $k \geq k_{0}(m)$. By Taubes' theorem [9], such an integer $k_{0}(m)$ exists. Moreover, with the above choices, we may even take $k_{0}(m)=0$ for $m<0, k_{0}(0)=6, k_{0}(1)=13$, and $k_{0}(2)=3[\mathbf{5}, \mathbf{6}, \mathbf{3}]$.

Now let $(m, n)$ be any pair of integers, and let $\tilde{n}$ be any integer such that

$$
\tilde{n} \leq \min \left(n-k_{0}(m)+\mathbf{c}_{\mathbf{1}}^{\mathbf{2}}\left(N_{m}\right), 2 n\right) .
$$

We may then define integers $k \geq k_{0}(m)$ and $\ell \geq 0$ by

$$
\begin{aligned}
k & =n-\tilde{n}+\mathbf{c}_{\mathbf{1}}^{\mathbf{2}}\left(N_{m}\right), \\
\ell & =2 n-\tilde{n} .
\end{aligned}
$$

Let $Z(k, m)$ be the twistor space of an anti-self-dual metric on $M=$ $N_{m} \# k \overline{\mathbb{C P}}_{2}$, and let

$$
X(k, \ell, m)=Z(k, m) \# \ell \mathbb{C P}_{3} .
$$

Notice that $X$ is a spin manifold. Moreover, it comes equipped with two different complex structures.

First, because $M=N_{m} \# k \overline{\mathbb{C P}}_{2}$ is a projective algebraic surface, $Z$ carries a projective algebraic complex structure $J_{1}$, and $X$ may then be identified with the blow-up of $\left(Z, J_{1}\right)$ at $\ell$ points. Let us denote this complex structure on $X$ by $J$. Using Proposition 2 and the above computations, we therefore have

$$
\begin{aligned}
\mathbf{c}_{\mathbf{1}}^{\mathbf{3}}(X, J) & =8(2 \chi+3 \tau)(M)+8 \ell=8 n \\
\mathbf{c}_{\mathbf{1}} \mathbf{c}_{\mathbf{2}}(X, J) & =6(\chi+\tau)(M)=24 m .
\end{aligned}
$$

On the other hand, each $Z$ also admits its twistor complex structure $J_{2}$, and we may instead choose to think of $X$ as the blow-up of this twistor space 
at $\ell$ points. Let us denote the corresponding complex structure on $X$ by $\tilde{J}$. Thus

$$
\begin{aligned}
\mathbf{c}_{\mathbf{1}}^{\mathbf{3}}(X, \tilde{J}) & =16(2 \chi+3 \tau)(M)+8 \ell=8 \tilde{n}, \\
\mathbf{c}_{1} \mathbf{c}_{\mathbf{2}}(X, \tilde{J}) & =12(\chi+\tau)(M)=48 m,
\end{aligned}
$$

as claimed.

Since the Todd genus of any complex 3 -fold is given by $\mathbf{c}_{1} \mathbf{c}_{2} / 24$, this result realizes all possible values of $\mathbf{c}_{\mathbf{1}} \mathbf{c}_{\mathbf{2}}$. The divisibility of $\mathbf{c}_{\mathbf{1}}^{\mathbf{3}}$ by 8 is also necessary for $X$ to be spin, so the result is also essentially optimal in this respect.

On the other hand, we have chosen to ignore $\mathbf{c}_{\mathbf{3}}$, which is determined by $(m, n, \tilde{n})$ in these examples. The abundance of rational curves also forces all our 3 -folds all have Kodaira dimension $-\infty$. And finally, most of our manifolds are in no sense minimal. It would obviously be of great interest to produce new examples which overcome these limitations.

Acknowledgments. The author would like to thank Ron Stern for drawing his attention to the problem and pointing out several important references. He would also like to the thank the Mathematics Department of Harvard University for its hospitality during the inception of this work.

\section{References}

[1] M. Atiyah, N. Hitchin and I. Singer, Self-duality in four dimensional Riemannian geometry, Proc. R. Soc. Lond., A 362 (1978), 425-461.

[2] E. Calabi, Construction and properties of some 6-dimensional almost complex manifolds, Trans. Amer. Math. Soc., 87 (1958), 407-438.

[3] S. Donaldson and R. Friedman, Connected sums of self-dual manifolds and deformations of singular spaces, Nonlinearity, 2 (1989), 197-239.

[4] N.J. Hitchin, Kählerian twistor spaces, Proc. Lond. Math. Soc., 43 (1981), 133-150.

[5] C. LeBrun, Scalar-flat Kähler metrics on blown-up ruled surfaces, J. Reine Angew. Math., 420 (1991), 161-177.

[6] C. LeBrun and M.A. Singer, A Kummer-type construction of self-dual 4-manifolds, Math. Ann., 300 (1994), 165-180.

[7] C. Okonek and A. Van de Ven, Cubic forms and complex 3-folds, Enseign. Math., 41(2) (1995), no. 3-4, 297-333.

[8] R. Penrose, Non-linear gravitons and curved twistor theory, Gen. Rel. Grav., 7 (1976), 31-52.

[9] C.H. Taubes, The existence of anti-self-dual metrics, J. Diff. Geom., 36 (1992), 163253.

[10] C.T.C. Wall, Classification problems in differential topology. V. On certain 6manifolds, Invent. Math., 1 (1966), 355-374; 2 (1966), 306. 
[11] S.-T. Yau, On the Ricci-curvature of a complex Kähler manifold and the complex Monge-Ampère equations, Comment. Pure Appl. Math., 31 (1978), 339-411.

Received February 19, 1998. This author was supported in part by NSF grant DMS9505744.

SUNY STONY BROOK

STONY BRoOK, NY 11794

E-mail address: claude@math.sunysb.edu 